\title{
Unpredictability and personality of cortical activity preceding action
}

\author{
Victor L Vvedensky* \\ RNC Kurchatov Institute, Moscow, Russia
}

\begin{abstract}
We analyzed magnetic signals of the brain during a second before voluntary finger movement. 3 of 18 our subjects displayed local alpha (10Hz) oscillations phasematched to the instant of the impending movement. Each subject had its own pattern of these oscillations. Alpha power and phase of oscillations were robustly linked to the instants of the finger movement selected at will, though large and unpredictable variations occurred permanently. We interpret our observations as a manifestation of the teamwork of several neural populations which reliably control preparation of the movement in each trial. Signals from a few members of the team are recorded during a particular trial - they show only a small fragment of the whole deterministic process. A new combination of active neurons is formed before each trial - this causes enhanced variability of measured signals. This variability is by no means a noise which should be removed from the data. Just the opposite, all these signals have to be used to reconstruct the sequence of events preparing the voluntary movement.
\end{abstract}

\section{Introduction}

Development of Brain-Computer Interfaces needs comprehensive analysis of the cortical activity preceding the intended action. However, "lack of understanding concerning the underlining neurophysiology continues to be the limiting factor in BCI development" [1]. Multichannel magnetic encephalography (MEG) is a highly appropriate tool for the study of the cortical activity during periods of time, when the subject is ready to act or move. No overt sign of impending movement can be seen before the action and the detected brain signals are not contaminated by the activity, evoked by the sensory system in response to the movement. Two approaches to study cortical processes in this time widow are currently used in electroencephalography (EEG) and MEG: Event Related Potentials/Fields (ERP, ERF) and Event Related Desynchronization/Synchronization (ERS, ERD). Both methods imply averaging of amplitude or power (squared amplitude) samples across many trials. The resulting average signals are considered to be descriptions of the neural processes related to the observed event. It is tacitly assumed that the same time course of the neural activation is basically present in each trial with, probably, some random variations. This should be confirmed. Each gradient sensor of our magnetometer is particularly selective to the activity of cortical sources located close to the sensor and picks up signals least contaminated by the rest of brain electrical activity. Many signals have characteristic shape (skewed triangle, for example, see Figures 1 and 2), which can be compared in different trials. We studied self-paced finger movements using a helmet-shaped whole head magnetometer [2] and did see that some components of brain response differ considerably between trials, being sometimes as high as background activity and sometimes virtually absent [3]. We have also found considerable variability of the magnetic signals before self-paced movement [4]. This variability did not look like a scatter around some basic behavior: the trials can be divided into groups with similar behavior inside each group. These findings provoked deeper scrutiny of the recorded signals from all our subjects.

The guidelines or recommendations for cognition studies [5] emphasize the need to carry out a comparison between a single case and a group of normal subjects. We see in our group of 18 normal subjects a number of single cases with spectacular behavior which deserve special analysis. The guidelines require the possibility to replicate the experiment. This severely limits the range of possible research. In our view many processes in the cortex resemble more the weather, which can hardly be reproduced at the request of the experimenter. The dynamics should be monitored on a single trial basis. We see that some processes do not replicate itself during subsequent portions of the same experimental run, though being reproducible inside considerable continuous time span. We highly appreciate the need of a set of guidelines in the cognition research, though they should not be centered on testing hypotheses and statistical analysis only. More sophisticated approaches are needed, which take into account unprecedented variability of the cognition related events in the brain. We cannot suggest suitable methods and techniques right now, though careful description of what we see in the brain during cognitive processing will probably lead to a proper approach to data analysis.

Experiments with self-paced movements are quite popular in the study of volition [6]. Average event related signal usually does not contain rhythmic components during a second before the movement, though high activity can be seen in individual trials. Spontaneous oscillations usually are not phase locked to the instant of the impending movement and are canceled by averaging. This is true for most of subjects, but not for all. Some subjects display robust peaks well before the movement occurs [4]. Amplitude of these peaks can be used for classification of oscillation patterns preceding the movement. At

Correspondence to: Victor L. Vvedensky, RNC Kurchatov Institute, Mitinskaya 17-2-460, 125222 Moscow, Russia, Tel: +7 495 7535961; E-mail: VictorLvo@ yandex.ru

Key words: voluntary movement, MEG, alpha oscillations preceding movement, inter- and intra-individual variability

Received: February 21, 2018; Accepted: March 12, 2018; Published: March 16, 2018 
least four of our subjects showed this behavior. Our analysis revealed another group of three subjects with much more spectacular and clearly visible behavior. Their event related responses contained considerable rhythmic component in the alpha range a second before the movement. Here we describe these phenomena in detail. This can be considered as the case study. A relatively small number of subjects, demonstrating this behavior, is not something out of the ordinary. Early studies revealed the presence of a rolandic wicket rhythm in just 18 persons out of 500 [7]. Now this phenomenon is called "motor mu rhythm" which occupies an appropriate place among the natural frequencies of the brain [8].

\section{Experimental Procedures}

Eighteen right-handed volunteers took part in the study-10 males and 8 females, 18-32 years old, two were left-handed. None of the participants had known neurological or psychiatric disorders. The study was approved by the local ethics committee of the Moscow University of Psychology and Education (MSUPE) and was conducted following the ethical principles regarding human experimentation (Helsinki Declaration).

The subjects were instructed to make quick index finger extensions at their own will, keeping the finger in the up position for some time and then moving it back into the original position. This series of events took about $3 \mathrm{~s}$, and the duration of each phase was chosen as convenient by the subject in each trial. Natural scatter in the duration was about $15 \%$, for some subjects much more. Each subject made more than 100 movements in a single session. The instant of the maximum acceleration of the fingertip was used as a reference point for the averaging of individual trials.

Magnetic brain responses were recorded with the sampling rate of $1000 \mathrm{~Hz}$ using a helmet-shaped whole head magnetometer (ElektaNeuromag MEG system, 306 channels). Native hardware filters with band pass $0.1-330 \mathrm{~Hz}$ were used and no additional filtering was applied to avoid distortion of the signal shape. During the measurement the participants were sitting in the magnetically shielded room. We processed signals from 204 channels measuring planar gradients of the magnetic field generated by the brain, since the main contribution to the gradient signal comes from the area located under the sensor. General data processing and calculation of the field patterns were done using the Brainstorm software [9]. The shape of the cortical surface was reconstructed using the FreeSurfer software [10].

\section{Results}

\section{Alpha oscillations phase matched to the impending movement}

We present observed phenomena individually for each subject, since they look different, though having common property - phase lock to the instant of the impending movement. Figure 1 illustrates our observations for Subject1. Sensors located over central (Rolandic) fissure in the left hemisphere detected triangular peak, which started to grow at the instant when right index finger moved with maximum acceleration. The signal grew to its maximum $50 \mathrm{~ms}$ later. The peak was clearly seen in several single trials and averaging of all 126 trials produced very neat shape - Figure 1A. Similar responses in the same area were recorded for all our subjects. Nearly identical peak shape is shown in Figure 2 for Subject 2. For majority of the subjects no rhythmic components were seen in the average signal in any sensor. However, for Subjectl the average signal displayed oscillations in the alpha frequency range (about $10 \mathrm{~Hz}$ ) in the sensors located over parieto-occipital sulcus of the right hemisphere - Figure 1A, B, C.
These oscillations were coherent during a second before the movement. Subject 1 chose the instant of the movement at will and the waiting time before the movement varied considerably for different trials (1.5 to $4.5 \mathrm{~s}$ ). In spite of this scatter, alpha oscillations before the movement were predominantly phase-matched to the instant of the impending movement. The processes in the distant cortical sites (Figure 1C) were coordinated well before the action become visible.

Similar relationship between finger movement, triangular peak in the sensory-motor area and preceding alpha oscillations were observed for Subject 2. In this case $50 \mathrm{~ms}$ peak and phase-locked alpha oscillations were recorded in the same sensor, as shown in Figure 2. It should be mentioned, that this behavior was again observed in the experiment with this subject three years later. The field maps indicate that the source of the recorded activity is located in the central fissure (primary sensory-motor area). Triangular peak was matched to the phase of alpha waves in the average signal, though clear dissociation between these processes was seen in individual trials - Figure 2A. All combinations (no alpha/peak, alpha/peak, alpha/no peak, no alpha/ no peak) were observed, which means that none of these signals was indispensable prerequisite for the finger movement in every run. We have to conclude that in addition to the triangular peak and alpha oscillations some other processes contribute to the movement generation. Maybe we can find them in other sensors and brain areas.

This peculiar behavior in individual trials prompted us to monitor individual events during the whole experimental session. In many neurophysiological studies, it is difficult to single out effects of individual events, since they usually follow each other closely. What is the correct way to distinguish the lasting effect of the previous event from the anticipation of the predictable next one in a raw? This is especially relevant for rhythmic phenomena lasting a fraction of a second. Figure 3 shows continuous records in two sensors (channels 443 and 1133) located symmetrically over the left and right hemispheres of Subject 2. This is about a quarter of the whole experimental run. The rest looks similar. We always see waxing and waning of the magnetic signal amplitude in different sensors. For alpha rhythm these variations are usually called spindles. All our subjects generate considerable number of spindles during the finger movement task performed with open eyes. It is generally accepted that the alpha rhythm is blocked when eyes are open, and our subjects were instructed to be visually attentive. Nevertheless we have seen a lot of oscillations in the alpha frequency range around $10 \mathrm{~Hz}$. Oscillations unresponsive to visual input are usually called mu-rhythm, though it is impossible to distinguish mu from alpha on-line. Typical record is shown in Figure $3 \mathrm{~B}$ for the channel 1133. Maximum amplitudes of the magnetic signal for most of our subjects are observed over left central and right parieto-occipital sulci (two areas shown in Figure 1C) and symmetric central sulcus in the right hemisphere. Long periods of calm, separating spindles, are frequently observed, sometimes lasting up to 20 seconds. One such period, 7 seconds long, is visible in Figure 3B.

For most of subjects and majority of sensors no temporal correlation between alpha power modulation and instants of the movements selected at will, were observed. However, when we monitor the channel with high rhythmic component in the average signal before finger movement, we see nearly perfect correspondence between variations of the alpha amplitude in this sensor and finger movements - Figure $3 \mathrm{~A}$, Subject 2. Positions of the alpha spindles inside waiting time period between movements indicate transient suppression of alpha waves by the short movement, with quick recovery after some delay. The suppression (or block) can be abrupt, as shown in Figure 2A for trials 

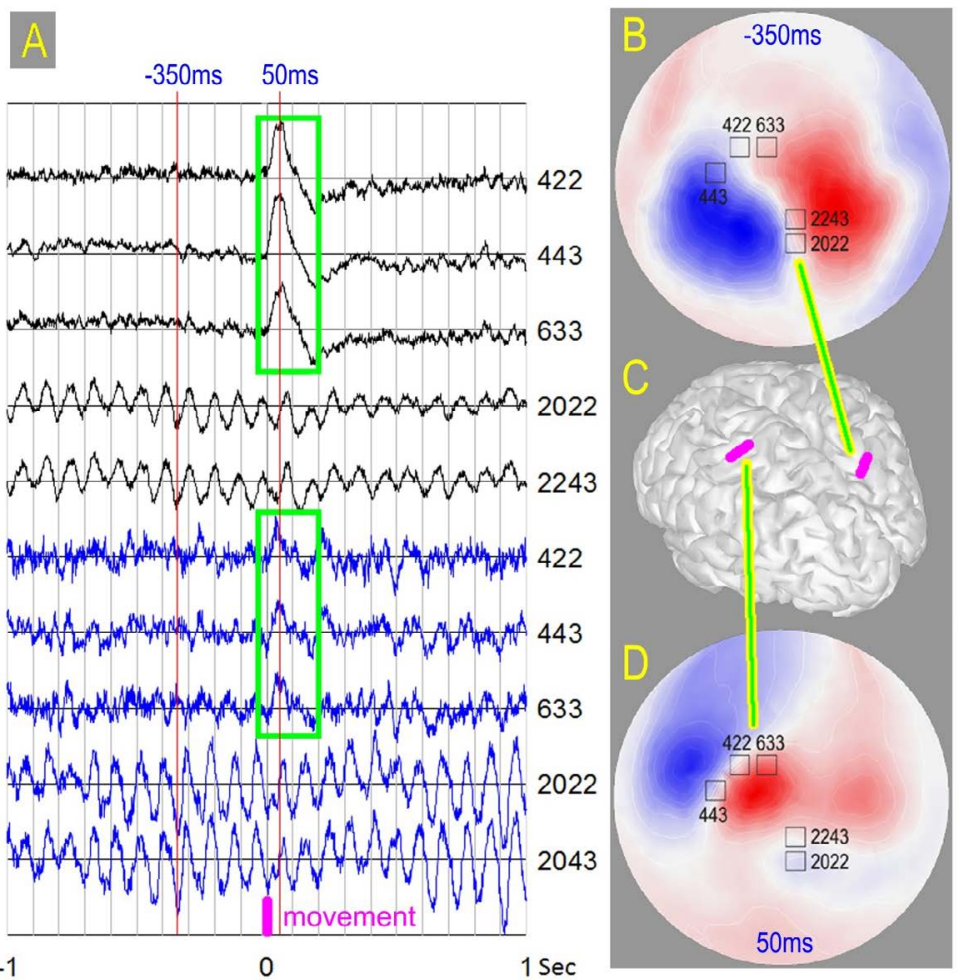

Figure 1. A) Maximum amplitude signals in five gradient sensors, average - black, single trial - blue. Sensor numbers are indicated for each trace. Sensor locations are shown on the maps $\mathrm{B}$ and D built over hemisphere covering the head. Self-paced quick finger extension occurs at the time instant zero. Maximum peak-to-peak amplitude for the single trial signal is $3 p \mathrm{~T}$, scale for the average signal is 5 times smaller. Green boxes highlight triangular peak with $50 \mathrm{~ms}$ delay, which looks similar for the average traces and this single trial traces. B) Field map for the average signal $350 \mathrm{~ms}$ before the finger movement (red line $-350 \mathrm{~ms}$ in A). This is the tip of the alpha wave preceding the movement. Maximum and minimum of the radial component of the magnetic field are shown in red and blue. Dipolar pattern implies cortical source in the parieto-occipital sulcus as shown in C. C) Positions of the electrical sources in the cortex of the Subject1 shown as magenta strips. Left strip corresponds to the map D, the right one to the map B. D) Field map for the average signal at the tip of the peak $50 \mathrm{~ms}$ after the finger movement. The instant is shown by the red line in A. Dipolar pattern implies cortical source in the Rolandic fissure as shown in C.
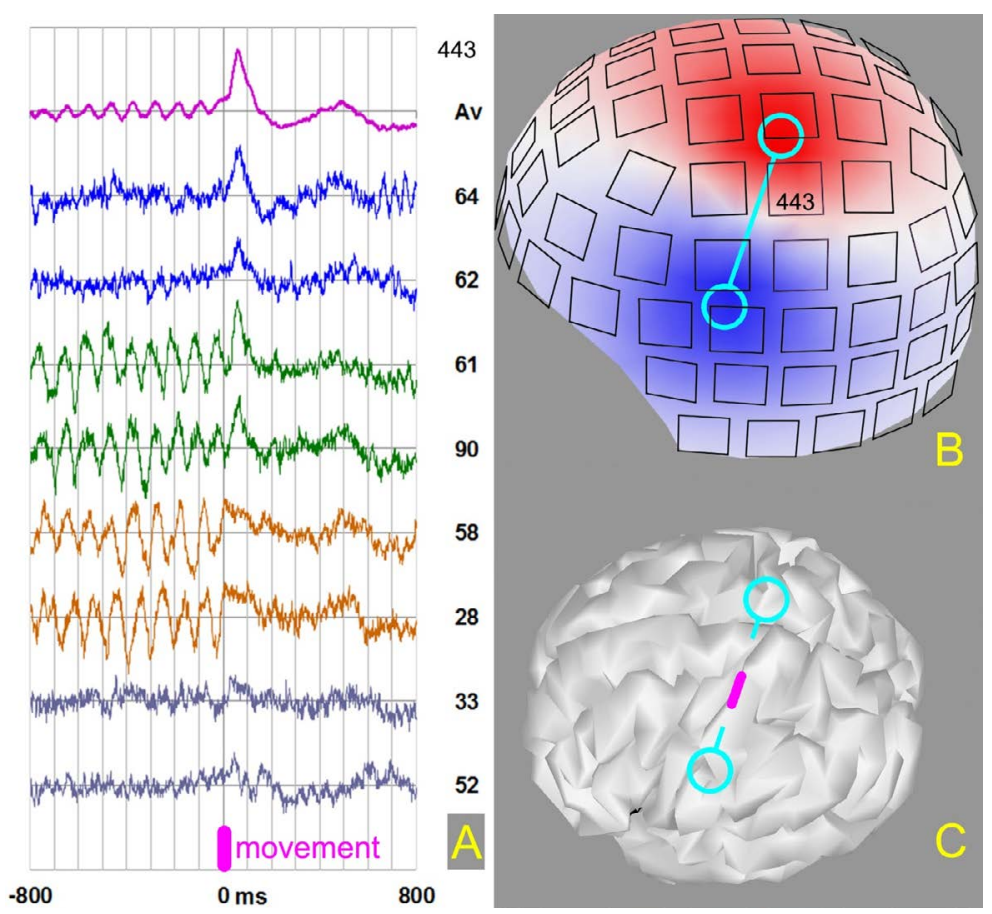

Figure 2. A) Magenta trace - average movement related signal in the channel 443 . Blue - trials 64 and 62 with no alpha component and high peak 50 ms after the finger extension. Green trials 61 and 90 with high alpha before movement and high peak. Sand - trials 58 and 28 displaying abrupt alpha block and no peak. Grey - trials 33 and 52 where no clear signals are visible. B) Map of the radial component of the magnetic field built over the surface of the helmet where locations of the sensors are indicated. Cyan dumbbell serves a view guide indicating positions of the field maxima for the peak. C) Center of the dumbbell points to the cortical area in the Rolandic fissure (highlighted in magenta) which generates the magnetic signal. 


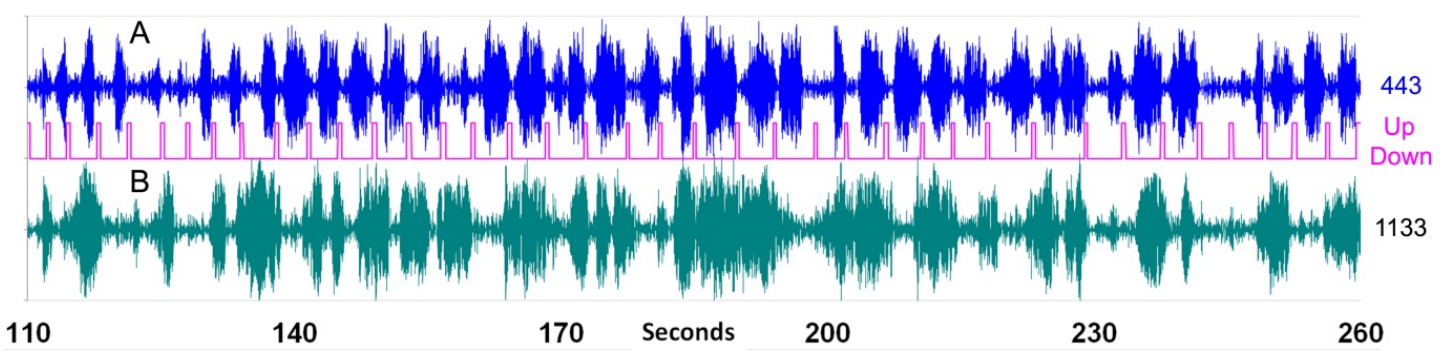

Figure 3. Magnetic field gradient changes in two sensors during 2.5 minutes of repetitive self-paced finger movements. Magenta curve indicates finger movements up and down. A) Blue curve is the band-path filtered (alpha range 6-14Hz) signal in the channel 443. It is the same sensor as in Figure 2. B) Green curve shows the band-path filtered signal in the channel 1133 , which is located over right hemisphere, symmetrically to the channel 443.

58 and 28. Sometimes alpha block occurs a couple of periods before the movement. Evolution of the alpha amplitude inside each cycle varies considerably. Some cycles do not contain alpha oscillations, though high spindles are seen both before and after this cycle. They are visible in Figure 3A. As mentioned above, alpha waves can be absent for tens of seconds and no modulation of alpha power by the movements can be seen during these periods of time.

Figure 3A displays robust dynamic link between cortical processes generating repetitive self-paced movements and electric oscillations in a small area on the cortical surface. This phenomenon can be clearly seen only in few gradient sensors out of 204 covering the head. It is highly reproducible during the whole experimental run 9 minutes long and this behavior has not changed in three years for this subject. However, this reproducibility is very peculiar. Scrutiny of each movement cycle reveals extremely high variability of the oscillation pattern during movement preparation. Both timing in respect to the movement instant and amplitude course vary considerably. Abrupt changes occur from cycle to cycle (trial to trial). For tens of seconds the effect can be invisible, since no alpha activity is recorded. Later on the modulation of the alpha amplitude with finger movements recovers.

\section{Discussion}

Our results can be considered as a demonstration of the fact that cortical oscillations can be reproducibly volitionally controlled during motor task. The topic of volitional control is extremely important for the development of Brain-Computer Interfaces [11]. Different parameters of cortical activity are considered for feedback. Sometimes quite complex measures like coherence of magnetic signals from different brain regions are analyzed [12]. Here we report a rare observation of a strong controlling parameter - quick changes of the amplitude of alpha $(\mathrm{mu})$ oscillations. We shall certainly proceed in finding control parameters for other subjects, though we would like to find out now what underlies phase-matching of rhythmic events to the impending movement for some of our subjects. This issue should be analyzed in depth.

We see a paradoxical situation: some cortical processes are robust (amplitude variations before movement and sharp peak after), though they occur or do not occur in unpredictable manner. We observe fine structure of evoked or induced alpha oscillations [13] in individual trials. When the trials are averaged this structure becomes blurred. Application of the averaging assumes repetition of the same cortical process is in every trial. This assumption is wrong in our case. The array of processes initiating the movement looks like a teamwork of several cortical populations each time conducting unexpected combination leading to a goal. Measuring device detects activity of only one or few members of the team. Before each movement we see only a small fragment of the complex pattern that reliably points the way to the goal - execution of the finger movement. We see no fault in any trial. Irregular alternation of different fragments is commonly called noise. The task is not to get rid of this noise, though to reconstruct the underlying pattern from a set of disparate pieces. This radically changes our view on the data processing needed to reconstruct sequences of events in the brain leading to overt action.

We need to consider available evidence on how actions are prepared in the systems of lower complexity. When individual animals are repeatedly observed in the same context they rarely behave in exactly the same way on every occasion [14]. A common fish displays a number (2-5) of different behavior patterns to leave shelter following a disturbance. It is unlikely that a large area in the human brain will exhibit more primitive behavior in response to the internal urge. On the other hand, simple averaging, implying stereotyped sequence of events in every trial, gives reliable evoked responses to external stimuli in many experimental settings. This discrepancy can be explained if internally or externally driven action serves an effective boundary between different types of cortical behavior. Experiments, using implanted multi-electrode arrays in the brain of macaque monkey, showed that stimulus onset drove a decline in across-trial firing-rate variability in multiple cortical areas [15]. This widespread variability decline suggests a rather general property of cortex, that its state is stabilized by an input. Our observations indicate that self-paced movement produces similar effect.

Before self-initiated action or perception of an external stimulus, cortical processes are much more variable then afterwards. This has important evolutionary background. Being an unpredictable prey is a good strategy to evade predators [16]. Predators spend time on observing their prey to improve their ability to capture more predictable prey over more unpredictable prey. Species with high within individual variability (unpredictability) and consistent variability across individuals (personality) is more likely to survive. This evidently holds for humans and we expect a number of different activity patterns for ensembles of cortical neurons preparing action unexpected for others. Such unpredictable actions are considered to be carried out at will. Huge inter-subject variability of volitional movements prevents a rapid deployment of BCI techniques for clinical and consumer applications [17]. Fortunately, the number of behaviors seems to be reasonable for an individual (7 to 10) [4]. This allows us to be optimistic about the possibility to predict the anticipated action based on measured brain signals. However, data processing should take into account both unpredictability and personality of underlying neural events in the brain.

Some internal changes cause instability of alpha spindles we see in Figure 3. Figure 3B shows variations unrelated to the self-paced movement, while Figure 3A displays nearly perfect fit, though with many unpredictable and large deviations. In our view continuous repetition 
of the same sequence of events in the standard neurophysiological experiment provokes spontaneous transitions between controlled an automatic execution of the task. This is like walking, which may require attention on rough surface and becomes automatic on a good road. We believe that these permanent transitions are reflected in the behavior of the cortical signals we detect with the magnetometer. Similar behavior was seen in experiments with rhesus monkeys directing their attention to one of two flashing stimuli [18]. Despite experimenter's best efforts attention fluctuated from trial to trial, and ability of monkey to detect a small change in a stimulus varied from $70 \%$ correct to nearly complete failure. However, responses of modestly sized populations of sensory neurons reliably predicted performance a short time later. A few dozen simultaneously recorded neurons gave the power to accurately predict behavior. Multichannel magnetometer provides opportunity to sample a subset of simultaneous cortical processes which are relevant to a particular impending action. One should develop appropriate detection scheme for the team of cortical ensembles performing the assigned task. The scheme has to be personalized and ready to deal with events never seen before.

\section{Conclusion}

Cortical processes before and after executed action differ considerably both in intra- individual variability (unpredictability) and inter-individual variability (personality). Our measurements of self-paced movements indicate that more flexible team of neural populations is involved in preparation of action, then in subsequent cortical processing. Apparent instability of behavior of these cortical ensembles is usually considered as noise, though it is not noise-like. We believe that measured brain signals reflect in any trial only a part of a robust neural process, which reliably controls the impending action. One should reconstruct the whole deterministic process from information provided by individual trials. Signal processing should take into account both personality of the subject and unpredictability of a voluntary action. Preliminary classification of subjects and trials is needed.

\section{Acknowledgment}

The author expresses his gratitude to A.O. Prokofyev, A.Yu. Nikolayeva, A.V. Butorina and T.A. Stroganova who provided their reliable experimental data. The research is supported by Russian Fund for Basic Research grant 15-29-03814-ofi_m.

\section{References}

1. Blakely T, Miller K, Ojemann JG, R.Rao R (2013) Exploring the Cortical Dynamics of Learning by Leveraging BCI Paradigms. In book: Brain-Computer Interface Research, Editors: (Guger Ch, Allison BZ, Edlinger G, eds), Springer-Verlag Berlin Heidelberg, pp: 53-60.

2. Chaianov NV, Prokof'ev AO, Morozov AA, Stroganova TA (2012) [Localization of cortical motor areas in humans by magnetoencephalography]. Zh Vyssh Nerv Deiat Im I P Pavlova 62: 629-640. [Crossref]

3. Vvedensky VL (2014) Individual trial-to-trial variability of different components of neuromagnetic signals associated with self-paced finger movements. Neurosci Lett 21 : 94-98. [Crossref]

4. Vvedensky VL, Prokofyev AO (2016) Timing of Cortical Events Preceding Voluntary Movement. Neural Comput 28: 286-304. [Crossref]

5. Picton TW, Bentin S, Berg P, Donchin E, Hillyard SA, et al. (2000) Guidelines for using human event-related potentials to study cognition: Recording standards and publication criteria. Psychophysiology 37: 127-152. [Crossref]

6. Hallett M (2007) Volitional control of movement: the physiology of free will. Clin Neurophysiol 118: 1179-1192. [Crossref]

7. Chatrian GE, Petersen MC, Lazarte JA (1959) The blocking of the roland wicket rhythm and some central changes related to movement. Electroencephalogr Clin Neurophysiol 11: 497-510. [Crossref]

8. Garcia-Rill E, D'Onofrio S, Luster B, Mahaffey S, Urbano FJ, et al. (2016) The $10 \mathrm{~Hz}$ Frequency: A Fulcrum For Transitional Brain States. Transl Brain Rhythm 1: 7-13. [Crossref]

9. Tadel F, Baillet S, Mosher JC, Pantazis D, Leahy RM (2011) Brainstorm: A UserFriendly Application for MEG/EEG Analysis. Computational Intelligence and Neuroscience.

10. Dale AM, Fischl B, Sereno MI (1999) Cortical surface-based analysis. I. Segmentation and surface reconstruction. Neuroimage 9: 179-194. [Crossref]

11. Fetz EE (2013) Volitional control of cortical oscillations and synchrony. Neuron 77 216-218. [Crossref]

12. Sacchet MD, Mellinger J, Sitaram R, Braun C, Birbaumer N, et al. (2012) Volitional control of neuromagnetic coherence. Front Neurosci 6: 189. [Crossref]

13. Pfurtscheller G (2003) Induced oscillations in the alpha band: functional meaning. Epilepsia 44 Suppl 12: 2-8. [Crossref]

14. Stamps JA, Briffa M, Biro PA (2012) Unpredictable animals: individual differences in intraindividual variability (IIV). Animal Behaviour 83: 1325-1334.

15. Churchland MM, Yu BM, Cunningham JP, Sugrue LP, Cohen MR, et al. (2010) Stimulus onset quenches neural variability: a widespread cortical phenomenon. Nat Neurosci 13: 369-378. [Crossref]

16. Chang CC, Teo HY, Norma-Rashid Y, Li D (2017) Predator personality and prey behavioural predictability jointly determine foraging performance. Sci Rep 7: 40734 .

17. van Gerven M, Farquhar J, Schaefer R, Vlek R, Geuze J, et al. (2009) The braincomputer interface cycle. J Neural Eng 6: 041001. [Crossref]

18. Cohen MR, Maunsell JHR (2010) A neuronal population measure of attention predicts behavioral performance on individual trials. J Neurosci 30: 15241-15253. [Crossref]

Copyright: (C2018 Vvedensky VL. This is an open-access article distributed under the terms of the Creative Commons Attribution License, which permits unrestricted use, distribution, and reproduction in any medium, provided the original author and source are credited. 